OPEN ACCESS

Edited by:

Shourong Shi,

Poultry Institute, Chinese Academy of Agricultural Sciences, China

Reviewed by:

Laura Glendinning,

University of Edinburgh,

United Kingdom

Jing Wang,

Feed Research Institute

(CAAS), China

${ }^{*}$ Correspondence:

Yu Yang

345605203@qq.com

Specialty section

This article was submitted to Animal Nutrition and Metabolism

a section of the journal

Frontiers in Veterinary Science

Received: 10 February 2021

Accepted: 28 May 2021

Published: 02 July 2021

Citation:

Sun B, Hou L and Yang Y (2021) The

Development of the Gut Microbiota and Short-Chain Fatty Acids of Layer Chickens in Different Growth Periods.

Front. Vet. Sci. 8:666535.

doi: 10.3389/fvets.2021.666535

\section{The Development of the Gut} Microbiota and Short-Chain Fatty Acids of Layer Chickens in Different Growth Periods

\author{
Baosheng Sun, Linyue Hou and Yu Yang* \\ Laboratory of Poultry Production, College of Animal Science, Shanxi Agricultural University, Jingzhong, China
}

A long-term observation of changes of the gut microbiota and its metabolites would be beneficial to improving the production performance of chickens. Given this, 1-day-old chickens were chosen in this study, with the aim of observing the development of the gut microbiota and gut microbial function using 16S rRNA gene sequencing and metabolites short-chain fatty acids (SCFAs) from 8 to 50 weeks. The results showed that the relative abundances of Firmicutes and genus Alistipes were higher and fiber-degradation bacteria were less at 8 weeks compared with 20 and 50 weeks $(P<0.05)$. Consistently, gut microbial function was enriched in ATP-binding cassette transporters, the energy metabolism pathway, and amino acid metabolism pathway at 8 weeks. In contrast, the abundance of Bacteroidetes and some SCFA-producing bacteria and fiber-degradation bacteria significantly increased at 20 and 50 weeks compared with 8 weeks $(P<0.05)$, and the two-component system, glycoside hydrolase and carbohydrate metabolism pathway, was significantly increased with age. The concentration of SCFAs in the cecum at 20 weeks was higher than at 8 weeks $(P<0.01)$, because the level of fiber and the number of dominant fiber-degradation bacteria and SCFA-producing bacteria were more those at 20 weeks. Notably, although operational taxonomic units (OTUs) and the gut microbial $\alpha$-diversity including Chao1 and abundance-based coverage estimator (ACE) were higher at 50 than 20 weeks $(P<0.01)$, the concentration of SCFAs at 50 weeks was lower than at 20 weeks $(P<0.01)$, suggesting that an overly high level of microbial diversity may not be beneficial to the production of SCFAs.

Keywords: gut microbiota, chicken, period, short-chain acid, functional prediction

\section{INTRODUCTION}

The gut microbiota play an important role in the poultry nutrition and health (1). Age is a dramatic factor that affects the microbial communities. The gut microbiota are distinct in different growth periods of chickens $(2,3)$. In the initial stage of colonization, facultative anaerobes were the principal bacteria, followed by strict anaerobes (4). Bacteroides and Eubacteria are established in 2 weeks, and the gut microbiota take 6-7 weeks to complete their establishment in chickens (5). The predominant phyla in the cecum are Firmicutes and Bacteroidetes over the whole life of chickens (6). Most of the current research on the succession of bacteria of chickens with age has focused on broilers (7-10). It was found that the colonization and function of the gut microbiota in broilers 
were different from 1 to 42 days $(11,12)$. In addition, some studies have focused on the microbiota of layer hens (13-16), the majority of which explored the short-term effect on the microbiota of young layers. In term of long-term observation, the gut microbiota in commercial Hy-Line layers from 1 to 51 weeks under field conditions were observed (17). A long-term development of cecal microbiota in egg-laying hens Lohmann Brown Light chickens from the day of hatching to 60 weeks old was also characterized (18). However, the above research on the layers' microbiome rarely observed the changes of intestinal microbiota function and microbial metabolites such as shortchain fatty acids (SCFAs) at the same time with age.

SCFAs, mainly including acetate, propionate, and butyrate, are derived from bacterial degradation and fermentation of dietary fibers. The cecum is the principal place for microbial fermentation of dietary fiber in chickens. Bacteroidetes is a kind of "generalist" that degrades dietary fiber polysaccharides. It can utilize a wide range of dietary polysaccharides from plants (19). Excellent fiber-degrading members of Bacteroidetes including Bacteroides (20) and Prevotella (21). Ruminococcus, Fibrobacter (22), Clostridium, and Roseburia (23) are excellent cellulolytic members of Firmicutes. SCFAs contribute to host nutrition and immune health (15). SCFAs can be used as energy and carbon source for poultry $(24,25)$. Acetate enters the liver for metabolism as a substrate for peripheral adipogenesis. Propionate reaches the liver as a substrate for gluconeogenesis. Butyrate serves as an energy source for colonic epithelial cells once SCFAs are absorbed. SCFAs can also regulate metabolism by inhibiting histone deacetylase (HDAC) and G protein-coupled receptors (GPCRs), such as GPR41 or GPR43 (26). In addition, SCFAs reduce intestinal $\mathrm{pH}$ (27) and induce the differentiation of regulatory $\mathrm{T}$ cells (28) to enhance the host health. SCFA production was also impacted by age (29).

Research on longitudinal observation of the ISA Brown layers' microbiome and SCFAs is lacking. Given this, ISA Brown Hens (IBH) were chosen in this experiment to observe the succession of the gut microbiota, enriched metabolic pathways, and SCFAs in different growth periods. Increasing our understanding of this would be beneficial to promoting production performance and the health of chickens by improving the gut microbiota and SCFAs.

\section{MATERIALS AND METHODS}

\section{Experimental Design and Animal Management}

This study was approved by the Shanxi Agricultural University Animal Experiment Ethics Committee (the license number: SXAU-EAW-2017-002Chi.001). In total, 108 1-day-old IBH were chosen. Chickens were randomly divided into nine replicates, with 12 chickens per replicate. Chickens were fed three different diets (Table 1) during brooding periods (0-8 weeks), growing periods (9-20 weeks), and laying periods (21-50 weeks).

Chickens were given free access to water and diet. The management of the temperature, light, and humidity was conducted according to the breeding manual. No conventional
TABLE 1 | Ingredients and nutrient levels of diets used in different growth periods.

\begin{tabular}{|c|c|c|c|}
\hline Item & $0-8$ weeks & 9-20 weeks & $21-50$ weeks \\
\hline \multicolumn{4}{|l|}{ Ingredients (\%) } \\
\hline Corn & 61.95 & 60.49 & 60.00 \\
\hline Soybean meal & 23.7 & 10 & 15.5 \\
\hline Bran & 0 & 8.5 & 0 \\
\hline Soybean oil & 1.1 & 0.5 & 0.6 \\
\hline Corn gluten meal & 4 & 0 & 1.6 \\
\hline Spray corn husk & 0 & 6.5 & 3.5 \\
\hline DDGS & 4 & 5.75 & 5 \\
\hline Peanut meal & 0 & 0 & 1 \\
\hline Stone power & 1.8 & 2.1 & 9.02 \\
\hline $\mathrm{CaHPO}_{4}$ & 1.3 & 0.7 & 0.65 \\
\hline $\mathrm{NaCl}$ & 0.3 & 0.28 & 0.25 \\
\hline Met & 0.2 & 0.06 & 0.14 \\
\hline Lys & 0.46 & 0.08 & 0.19 \\
\hline Thr & 0.09 & 0.04 & 0 \\
\hline Multivitamin $^{a}$ & 0.4 & 0.35 & 0.4 \\
\hline Minerals ${ }^{b}$ & 0.55 & 0.5 & 0.45 \\
\hline Zeolite & 0 & 2 & 0.5 \\
\hline Choline chloride & 0.1 & 0.05 & 0.1 \\
\hline Complex enzyme & 0.05 & 0 & 0 \\
\hline Monosodium glutamate & 0 & 2 & 1 \\
\hline Protein powder & 0 & 0.1 & 0.1 \\
\hline Total & 100 & 100 & 100 \\
\hline \multicolumn{4}{|l|}{ Nutrient levels ${ }^{c}$} \\
\hline ME (MJ/kg) & 12.43 & 11.50 & 10.62 \\
\hline Crude protein (\%) & 19.49 & 15.3 & 16 \\
\hline Crude fiber (\%) & 3.21 & 3.95 & 2.95 \\
\hline Crude fat (\%) & 4.27 & 3.99 & 3.72 \\
\hline Crude ash (\%) & 5.83 & 5.67 & 12.13 \\
\hline $\mathrm{Ca}(\%)$ & 1.05 & 0.99 & 3.55 \\
\hline Total P (\%) & 0.57 & 0.5 & 0.43 \\
\hline $\mathrm{NaCl}(\%)$ & 0.3 & 0.37 & 0.31 \\
\hline
\end{tabular}

DDGS, dried distiller's grain with solubles.

${ }^{a} 0-8$ weeks: per kilogram of diet contained vitamin A 2,100-2,500 KIU; vitamin B1 $\geq 620 \mathrm{mg}$; vitamin $B 2 \geq 1,600 \mathrm{mg}$; vitamin $B 5 \geq 2,450 \mathrm{mg}$; vitamin $B 6 \geq 830 \mathrm{mg}$; niacinamide $\geq 7,000 \mathrm{mg}$; vitamin $B 12 \geq 4,200 \mu \mathrm{g}$; vitamin D3 800-1,240 KIU; vitamin $E \geq 5,900$ IU; vitamin $K 3 \geq 600 \mathrm{mg}$; folic acid $\geq 245 \mathrm{mg}$; biotin $\geq 35 \mathrm{mg}$. 9-20 weeks: vitamin A 2,150-3,250 KIU; vitamin B1 $\geq 440 \mathrm{mg}$; vitamin $B 2 \geq 1,280 \mathrm{mg}$; vitamin B5 $\geq 2,140 \mathrm{mg}$; vitamin $B 6 \geq 640 \mathrm{mg}$; vitamin $B 12 \geq 3,200 \mathrm{mg}$; vitamin $D 3$ 550-1,650 KIU; vitamin $E \geq 4,260$ IU; vitamin $K 3 \geq 430 \mathrm{mg}$; nicotinamide $\geq 6,400 \mathrm{mg}$; folic acid $\geq 280 \mathrm{mg}$; biotin $\geq 40 \mathrm{mg}$. 21-50 weeks: vitamin A 145 to $190 \mathrm{KIU}$; vitamin B1 $\geq 26 \mathrm{mg}$; vitamin B2 $\geq 100 \mathrm{mg}$; vitamin $B 5 \geq 160 \mathrm{mg}$; vitamin $B 6 \geq 60 \mathrm{mg}$; vitamin $B 12 \geq 200 \mathrm{mg}$; vitamin $D 3$ 30 to $95 \mathrm{KIU}$; vitamin $E \geq 355 \mathrm{IU}$; vitamin $K 3 \geq 50 \mathrm{mg}$; nicotinamide $\geq 552 \mathrm{mg}$; folic acid $\geq 12 \mathrm{mg}$; biotin $\geq 2 \mathrm{mg}$; choline chloride $\geq 6.5 \mathrm{mg}$.

${ }^{b} \mathrm{O}-8$ weeks: per kilogram of diet contained $\mathrm{Cu} 8 \mathrm{mg}, \mathrm{Fe} 80 \mathrm{mg}, \mathrm{Me} 60 \mathrm{mg}$, Se $0.15 \mathrm{mg}$, Zn 40 mg, I $0.35 \mathrm{mg}$. 9-20 weeks: Fe 1,300-7,400 mg; Cu 120-650 mg; Mn 1,4502,900 mg; Zn 1,250-2,900 mg; I 7-95 mg; Se 6-9.5\%; Ca 12-25\%; P (adding phytase) $\geq 2.0 \%$; NaCl 4-10\%; methionine $\geq 1.8 \%$; moisture $\leq 10 \%$. 21-50 weeks: Fe 1,3007,400 mg; Cu 120-650 mg; Mn 1,450-2,900 mg; Zn 1,250-2,900 mg; I 8-95 mg; Se 6-9.5\%; Ca 12-25\%; P (adding phytase) $\geq 2.1 \%$; NaCl 4-10\%; methionine $\geq 2.3 \%$; moisture $\leq 10 \%$.

${ }^{c}$ The composition was calculated but not measured for the diet used.

immunization schedule was performed to avoid impacts on the gut microbiota. 


\section{Sample Collection}

A chicken from each replicate was chosen at the end of 8 , 20 , and 50 weeks. They were slaughtered humanely using oral bloodletting slaughtering method. The contents of the left cecum per bird were collected into multiple cryogenic tubes, and they were put into a liquid nitrogen tank and then preserved at $-80^{\circ} \mathrm{C}$ until the 16S rRNA gene sequence of the gut microbiota and the determination of the concentration of SCFAs.

\section{Sample Determination 16S rRNA Gene Sequencing of Gut Microbiota}

It was sequenced by Genedenovo Biotechnology Ltd. (Guangzhou, China) using High-Throughput Sequencing Technology. First, DNA extraction was performed using the HiPure Stool DNA Kits (Guangzhou, China). V3-V4 regions of the $16 \mathrm{~S}$ rRNA gene were amplified by PCR using primers 341F $5^{\prime}$-CCTACGGGNGGCWGCAG and 806R $3^{\prime}$ GGACTACHVGGGTATCTAAT. The barcode is an eight-base sequence unique to each sample. PCRs were as follows: $95^{\circ} \mathrm{C}$ for $2 \mathrm{~min}$, followed by 27 cycles at $98^{\circ} \mathrm{C}$ for $10 \mathrm{~s}, 62^{\circ} \mathrm{C}$ for $30 \mathrm{~s}$, $68^{\circ} \mathrm{C}$ for $30 \mathrm{~s}$, and a final extension at $68^{\circ} \mathrm{C}$ for $10 \mathrm{~min}$. PCRs were performed in triplicate with $50 \mu \mathrm{l}$ of mixture containing 5 $\mu \mathrm{l}$ of $10 \times$ KOD Buffer, $5 \mu \mathrm{l}$ of $2.5 \mathrm{mM}$ of dNTPs, $1.5 \mu \mathrm{l}$ of each primer, $1 \mu \mathrm{l}$ of KOD Polymerase, and $100 \mathrm{ng}$ of template DNA.

Illumina Hiseq 2500 (Illumina, Inc., San Diego, CA, USA) sequencing was then performed. Amplicons were extracted from $2 \%$ agarose gels and purified using the AxyPrep DNA Gel Extraction Kit (Axygen Biosciences, Union City, CA, USA) and quantified using ABI Ste1OnePlus Real-Time PCR System (Life Technologies, Carlsbad, CA, USA). Purified amplicons were pooled in equimolar and paired-end sequenced $(2 \times 250)$ on an Illumina platform. The datasets presented in this study can be found in online repositories. The names of the repository and accession number can be found in the National Center for Biotechnology Information (NCBI) Sequence Read Archive (SRA), PRJNA701972.

\section{Bioinformatics Analysis \\ Quality Control and Read Assembly}

Raw reads were further filtered using FASTP. Paired-end clean reads were merged as raw tags using FLSAH (30) (version 1.2.11) with a minimum overlap of $10 \mathrm{bp}$ and mismatch error rates of $2 \%$. Raw tag filtering noisy sequences of raw tags were filtered by QIIME (31) (version 1.9.1) pipeline under specific filtering conditions (32) to obtain the high-quality clean tags. Chimera checking and removal: Clean tags were searched against the reference database to perform reference-based chimera checking using UCHIME algorithm. All chimeric tags were removed, and the final obtained effective tags were used for further analysis.

\section{Operational Taxonomic Unit Cluster}

Effective tags were clustered into operational taxonomic units (OTUs) with 97\% similarity using the UPARSE pipeline (33). The tag sequence with the highest abundance was selected as a representative sequence within each cluster. Venn analysis was performed in $\mathrm{R}$ project (version 3.4.1) to identify unique and common OTUs.

\section{Taxonomy Classification}

The representative sequences were classified into organisms using the Ribosomal Database Project classifier (version 2.2) (34) based on SILVA database (35) with the confidence threshold values ranging from 0.8 to 1 . The abundance statistics of each taxonomy were visualized using Krona (36) (version 2.6).

\section{Microbial Diversity Analysis}

$\alpha$-Diversity indices including abundance-based coverage estimator (ACE), Chao1, Shannon, and Simpson were calculated in QIIME. The comparison of $\alpha$-diversity indices among groups was performed by the Kruskal-Wallis using Vegan package in $\mathrm{R}$ project (37). $\beta$-Diversity was performed. Sequence alignment was performed using Muscle (38) (version 3.8.31), and then weighted uniFrac distance matrix was generated by GuniFrac package (version 1.0) in R project.

\section{Function Prediction}

Functional profiles including Kyoto Encyclopedia of Genes and Genomes (KEGG) Orthology (KO) and enriched metabolism pathways of OTUs were inferred using a software package Tax4Fun (39). Microbiome phenotypes of bacteria were classified using BugBase. FAPROTAX database (Functional Annotation of Prokaryotic Taxa) and associated software (version 1.0) were used for generating the ecological functional profiles of bacteria. Heatmaps were made by $\mathrm{R}$ pheatmap package. The predicted $\mathrm{KO}$ and ko abundances were normalized by $Z$-score and then plotted.

\section{Determination of the Concentration of Short-Chain Fatty Acids}

The concentration of SCFAs ( $\mathrm{mmol} / 100 \mathrm{~g}$ ) in the cecum chyme was measured using the internal standard method with High Performance Gas Chromatography (Trace 1300, Thermo Fisher Scientific, Waltham, MA, USA) (40).

First, a solution containing internal standard crotonic acid was prepared. Metaphosphoric acid $25 \mathrm{~g}$ and crotonic acid $0.6464 \mathrm{~g}$ were accurately weighed, and they were put into a $100-\mathrm{ml}$ volumetric flask and up to $100 \mathrm{ml}$ with ultrapure water. Then, $100 \mathrm{ml}$ of mixed standard stock solutions was prepared as follows: different volumes of standards were added (Table 2) into a $100-\mathrm{ml}$ volumetric flask, topped up to $100 \mathrm{ml}$ with ultrapure water, and preserved at $4^{\circ} \mathrm{C}$. The concentration $(\mathrm{g} / \mathrm{L})$ of additive was calculated according to the density of each standard (e.g., acetate is $1.050 \mathrm{~g} / \mathrm{ml}$ ), and then it was converted into the mol concentration $(\mathrm{mmol} / \mathrm{L})$ based on molar mass of each standard (e.g., acetate is $60 \mathrm{~mol} / \mathrm{g}$ ). The volatile fatty acid standard solution was prepared as follows: $0.2 \mathrm{ml}$ of deproteinized metaphosphate solution containing crotonic acid was added to three $1.5-\mathrm{ml}$ centrifuge tubes, and $1 \mathrm{ml}$ of mixed standard stock solution was added to this. The peak area of crotonic acid in the standard solution was measured.

Sample preparation: 0.5 - to $1-\mathrm{g}$ contents of the cecum were added to nine times the weight of ultrapure water, homogenate, and centrifuged at $10,000 \mathrm{rpm}$ for $10 \mathrm{~min}$, and the supernatant was removed. Then, $1 \mathrm{ml}$ of supernatant sample was placed into a 1.5-ml Eppendorf (EP) tube, and $0.2 \mathrm{ml}$ of mixed solution of crotonic metaphosphate was 
TABLE 2 | The additive volume and concentrations of volatile fatty acid standards added to the standard stored solution.

\begin{tabular}{|c|c|c|c|c|c|c|}
\hline & Acetate & Propionate & Butyrate & Isobutyrate & Isovalerate & Valerate \\
\hline Additive volume $(\mu l)$ & 60 & 40 & 20 & 5 & 5 & 5 \\
\hline Concentration $^{\mathrm{a}}(\mathrm{g} / \mathrm{L})$ & 0.63 & 0.40 & 0.19 & 0.048 & 0.047 & 0.047 \\
\hline Mol concentration ${ }^{\mathrm{b}}(\mathrm{mmol} / \mathrm{L})$ & 10.50 & 5.35 & 2.19 & 0.54 & 0.46 & 0.46 \\
\hline
\end{tabular}

${ }^{a}$ Concentration of additive standards $(\mathrm{g} / \mathrm{L})=$ density of standards $(\mathrm{g} / \mathrm{mL}) \times$ additive volume $(\mu /) \div 100$.

${ }^{b} \mathrm{Mol}$ concentration $(\mathrm{mmol} / \mathrm{L})=$ concentration of additive standard $(\mathrm{g} / \mathrm{L}) \div$ molar mass of standard $(\mathrm{g} / \mathrm{mol}) \times 1,000$.

added and reacted for $3 \mathrm{~h}$. Centrifugation at $12,000 \mathrm{r}$ for 5 min was undertaken. The supernatant was injected into the chromatograph instantaneously with a $10-\mu 1$ microinjector, and the injection volume was $1.0 \mu \mathrm{l}$. Reaction conditions were set as follows: injection temperature $220^{\circ} \mathrm{C}$; initial temperature $70^{\circ} \mathrm{C}$; detector temperature $220^{\circ} \mathrm{C}$; split 5; split ratio 6; constant current $0.8 \mathrm{ml} / \mathrm{min}$; tail blowing $40 \mathrm{ml} / \mathrm{min}$; and hydrogen $35 \mathrm{ml} / \mathrm{min}$ and air $350 \mathrm{ml} / \mathrm{min}$.

The concentration of a certain acid $(\mathrm{mmol} / \mathrm{L})=($ peak area of certain acid of sample $\times$ peak area of crotonic acid in standard solution $\times$ mol concentration of certain acid) $\div$ (peak area of crotonic acid in sample $\times$ peak area of certain acid in standard solution).

\section{Statistical Analysis}

In terms of gut microbiota, the comparisons of the relative abundance phyla and genera in groups were performed by Metastats (41) (version 20090414). Metastats showed significantly different relative abundances of bacteria using $P$ $<0.01$ or 0.05 . Multivariate statistical techniques including principal component analysis (PCA), principal coordinates analysis (PCoA), and non-metric multidimensional scaling (NMDS) of weighted uniFrac distances were calculated and plotted in R project. Statistical analysis of Welch's $t$-test and Anosim test was calculated using $\mathrm{R}$ project. The $\beta$-diversity analyses between groups were calculated by the Kruskal-Wallis using Vegan package in $\mathrm{R}$ project. Heatmap analysis was performed using the $\mathrm{R}$ package. Analysis of function difference between groups was calculated by Welch's $t$-test in $\mathrm{R}$ package (version 2.5.3). Statistical analyses of SCFAs were performed using a one-way ANOVA with SPSS 22.0 software. The results are expressed as the means and standard error of the mean (SEM).

\section{RESULTS}

\section{Operational Taxonomic Units and Microbial Diversity of Chickens in Different Periods}

For simplicity, "ISA Brown Hens- 8 weeks" was named IBHE for short, "ISA Brown Hens-20 weeks" was named IBHT, and "ISA Brown Hens-50 weeks" was named IBHF.

The total and unique numbers of OTUs at 50 weeks (1,629 and 823 ) were more than those at 8 (963 and 193) and 20 weeks (958 and 144) (Figure 1). Moreover, the $\alpha$-diversity indices including ACE and Chaol significantly increased with age $(P<0.01)$ (Table 3). It is suggested that the microbial community richness significantly increased with age. The gut microbial diversity of

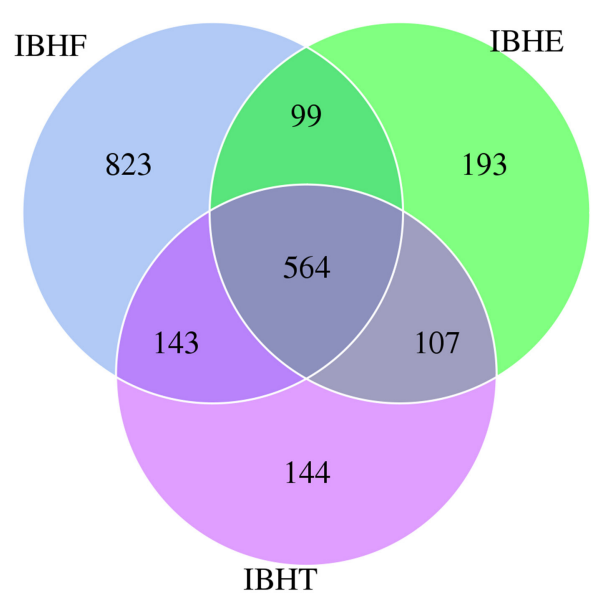

FIGURE 1 | Venn diagram of operational taxonomic units (OTUs) in different growth periods. Note that the overlapping parts show the OTUs shared by three groups, and the numbers on both sides are the unique OTUs owned by each group. IBHE, IBHT, and IBHF refer to "ISA Brown Hens-8 weeks," "ISA Brown Hens-20 weeks," and "ISA Brown Hens-50 weeks," respectively.

IBHF was the highest among three periods (Table 3); this was also consistent with the number of OTUs.

For the $\beta$-diversity indices, the PCA (Figure 2A) and PCoA (Figure 2B) showed that the samples were separated in the first principal component. NMDS showed that the stress $=0.091$ $<0.1$, indicating that the accuracy of the model was good (Figure 2C). In addition, Anosim test showed that $\mathrm{R}=1>$ 0 , indicating that the difference of microorganisms between groups was greater than that within groups; however, $P=0.1$ $>0.05$ represented that there was no significant difference in the pairwise comparison of three periods (Figure 2D). On the whole, the samples were clustered by different growth periods, but there was no significant difference between different periods $(P>0.05)$.

\section{Gut Microbial Composition in Different Growth Periods}

At the phylum level (Figure 3A), the dominant phyla of all samples were both Firmicutes and Bacteroidetes. The abundance of "generalist" Bacteroidetes (44.40, 62.55, and $73.65 \%$ ) (Figure 3B) significantly increased at 8, 20, and 50 weeks, respectively $(P<0.01)$, but Firmicutes $(40.47 \%, 23.41$, and $16.76 \%$ ) (Figure 3C) and Proteobacteria (7.27, 3.37, and $2.70 \%)$ 
TABLE 3 | Comparison of $\alpha$-diversity indices among different growth periods.

\begin{tabular}{|c|c|c|c|c|c|}
\hline Diversity indices & 8 weeks & 20 weeks & 50 weeks & SEM & $P$-value \\
\hline ACE & $1,594.39^{\mathrm{Bc}}$ & $1,653.17^{\mathrm{Bb}}$ & $2,460.21^{\mathrm{Aa}}$ & 168.13 & 0.0010 \\
\hline Chao1 & $1,547.78^{\mathrm{Bc}}$ & $1,660.92^{\mathrm{Bb}}$ & $2,422 \cdot 61^{\mathrm{Aa}}$ & 134.25 & 0.0070 \\
\hline Shannon & 6.54 & 6.51 & 7.16 & 0.26 & 0.017 \\
\hline Simpson & 0.95 & 0.97 & 0.98 & 0.0047 & 0.56 \\
\hline
\end{tabular}

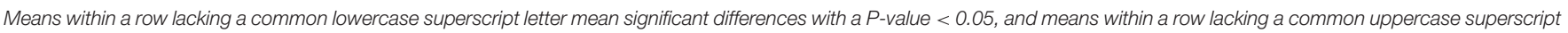
letter mean extremely significant differences with a P-value $<0.01$. Data are expressed as the means and pooled standard error of the mean (SEM).

ACE, abundance-based coverage estimator.

(Figure 3D) significantly decreased with age $(P<0.01)$. Special cellulose-degradation phylum Fibrobacteres was only found at 50 weeks $(P<0.01)$ (Figure 3E).

At the genus level (Figure 4A), the dominant genus of all samples was Bacteroides $(27.97,24.84$, and $26.29 \%)(P>0.05)$. The dominant bacteria at 8 weeks included the bile-tolerant bacterium Alistipes (7.38\%) (Figure 4B) and low-abundance butyrate-producing genus Anaerostipes (0.4\%) (Figure 5A) compared with 20 weeks (1.23\%) (0.033) and 50 weeks $(0.76 \%)$ (0.00021) $(P<0.01)$, respectively. In addition, Bacteroides thetaiotaomicron $(0.006 \%)$, which is one of the best fiberdegrading species, was only detected at 8 weeks (Figure 5B). The dominant bacteria genera at 20 and 50 weeks included Rikenellaceae_RC9_gut_group (14.56 and 15.23\%) $(P<0.01)$ (Figure 4C), propionate-producing genus Phascolarctobacterium (1.92 and 2.76\%) $(P<0.01)$ (Figure 4E), fiber-degradation bacteria Prevotellae_UCG_001 (1.81 and 2.13\%) $(P<0.01)$ (Figure 4D), and Alloprevotella $(0.56$ and $0.92 \%)(P<0.05)$ (Figure 4F) compared with those at 8 weeks $(1.09,0.80,0.26$, and $0.21 \%)$, respectively.

The dominant bacteria at 20 weeks also included lowabundance fiber-degradation and acetate-producing genus Bifidobacterium (0.028\%) (Figure 5C) and potential fiberdegradation bacteria Prevotellae_Ga6A1_group (0.62\%) (Figure 5D) compared with those at 8 and 50 weeks. Bifidobacterium was also a famous probiotic genus. The dominant bacteria at 20 weeks also included some lactateproducing species such as Lactobacillus aviaries (0.014\%), Lactobacillus agilis (0.029\%), and Lactobacillus alvi (0.043\%) (Figure 5E). Moreover, many unique fiber-degradation bacteria including Fibrobacter (0.00072\%), Prevotellae_UCG_003 (0.13\%), Prevotella_1 (0.11\%), and Prevotella_9 (0.0026\%) were only found at 50 weeks (Figures 5F,G).

\section{Functional Prediction of the Gut Microbiota in Different Growth Periods}

The KO of OTUs was further predicted (Figure 6A). The heatmap showed that ATP-binding cassette $(\mathrm{ABC})$ transports including $\mathrm{K} 06147(0.0066,0.0063$, and $0.0056 \%), \mathrm{K} 02003$ (0.0042, 0.0039, and $0.0036 \%)$, and $\mathrm{K} 01990$ (0.0031, 0.0030, and $0.0027 \%)$ significantly decreased at 8,20 , and 50 weeks $(P$ $<0.05$ ) (Figure 6B), respectively. In contrast, $\beta$-galactosidase K01190 (0.0039, 0.0050, and 0.0056\%), $\beta$-glucosidase K05349 $(0.0034,0.0042$, and $0.0051 \%)$, and two-component system (TCS) including $\mathrm{K} 00936$ (0.0060, 0.0071, and 0.0075\%) and K07636 $(0.0029,0.0035$, and $0.0039 \%)$ significantly increased with age $(P$ $<0.01$ ) (Figure 6C).

Then, the enriched metabolic pathways of OTUs were predicted (Figure 7A). Amino acid metabolism pathways ko00330 (0.021, 0.019, and $0.018 \%)$, the energy metabolism pathway ko00190 $(0.017,0.016$, and $0.015 \%)$, and the membrane transport of ABC transporters ko02010 (0.075, 0.066, and $0.029 \%)$ significantly decreased at 8,20 , and 50 weeks $(P<0.01)$ (Figure 7B), respectively. In contract, carbohydrate metabolism pathways ko00500 (0.026, 0.027, and 0.029\%) and ko00051 $(0.017,0.021$, and $0.021 \%)(P<0.01)$ and the TCS pathway ko02020 $(0.068,0.070$, and $0.072 \%)(P<0.05)$ significantly increased with age. In addition, the carbohydrate metabolism pathway ko00051 (0.021 and 0.021\%) was enriched at 20 and 50 weeks compared with 8 weeks $(0.017 \%)(P<0.01)$ (Figure $7 \mathrm{C})$.

\section{Concentration of Short-Chain Fatty Acids in Different Growth Periods}

Next, the concentration of gut microbial metabolites SCFAs was measured (Table 4). The concentration of SCFAs at 20 weeks was higher than that at 8 weeks $(P<0.01)$. Interestingly, although the microbial diversity at 50 weeks was the highest, the concentration of SCFAs at 20 weeks was also almost twice as high as that at 50 weeks $(P<0.01)$.

\section{DISCUSSION}

\section{Changes of Microbial Diversity in Different Growth Periods}

The gastrointestinal tract of newly hatched chickens immediately has microbial colonization (42). With the growth of age, the cecal microorganisms form a complex community (43). In this experiment, the numbers of OTUs and gut microbial $\alpha$-diversity including ACE and Chaol of chickens significantly also increased with age. The larger the value of ACE and Chaol, the higher the community richness. This is consistent with previous reports that increasing taxonomic richness and diversity were observed in chickens through time (9). The $\alpha$-diversity of the gut microbiota of pigs also increased with age (21). In this experiment, the $\beta$ diversity of the samples was clustered by different growth periods, but NMDS analysis showed the difference between different periods was not great. It was consistent with a report that NMDS showed that 0 - to 42-day-old chicken gut microbiota could 

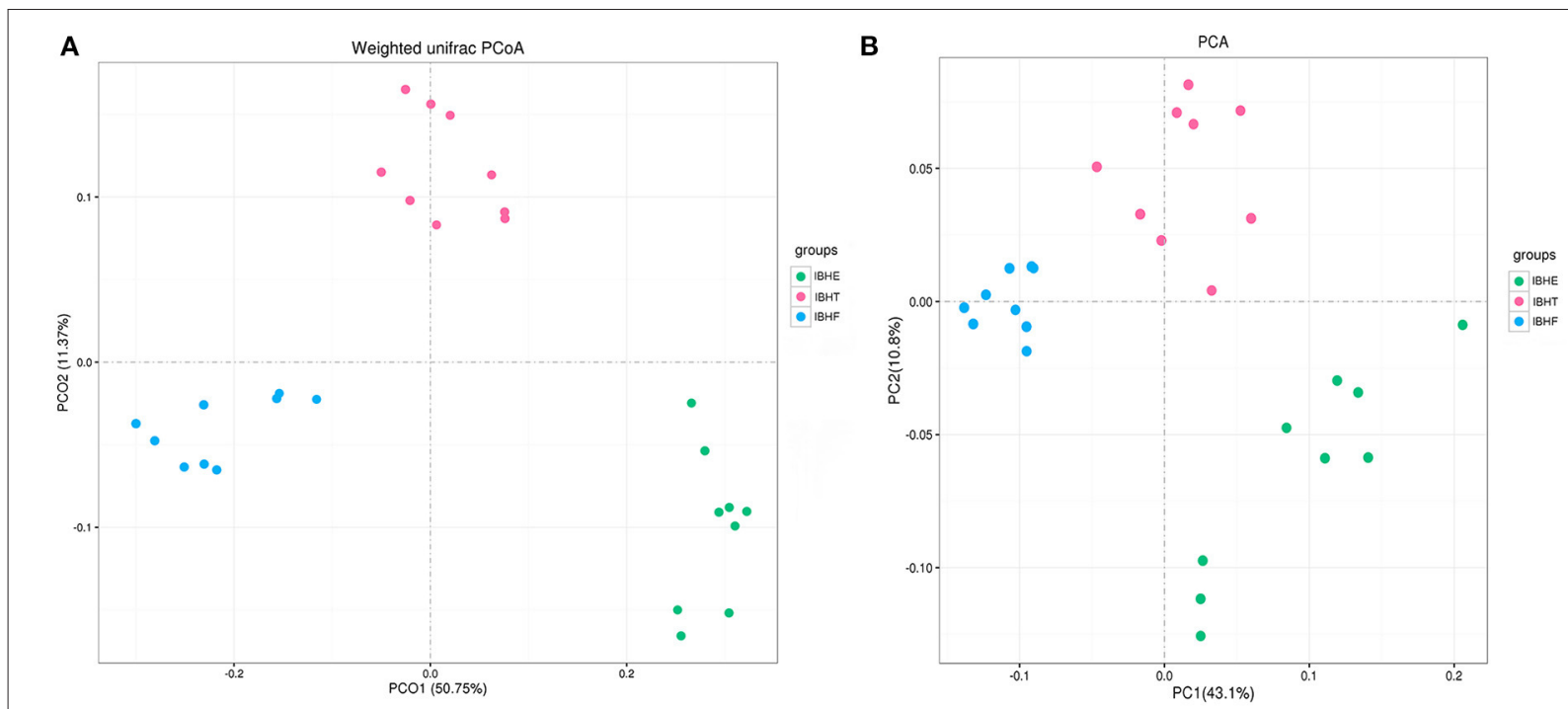

C

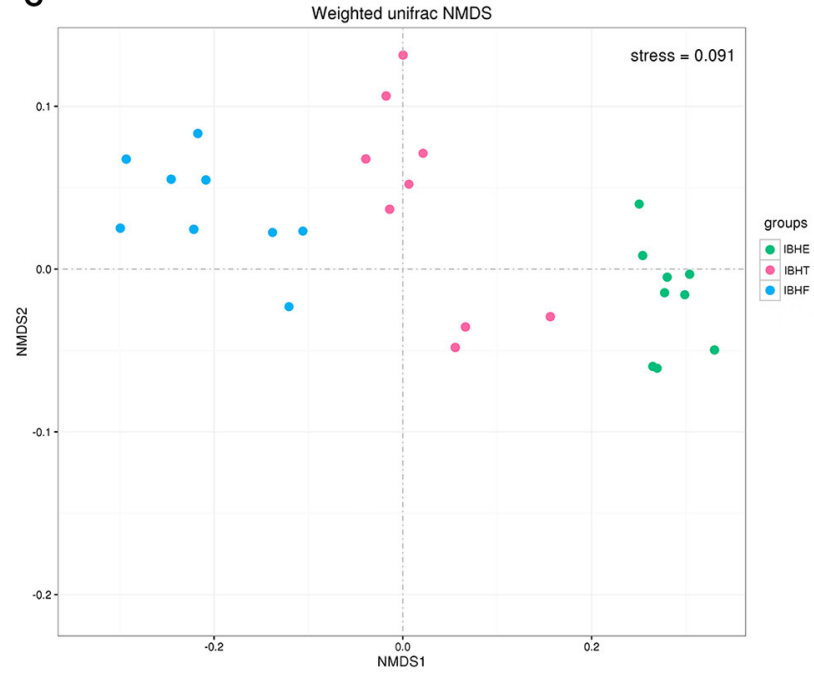

D

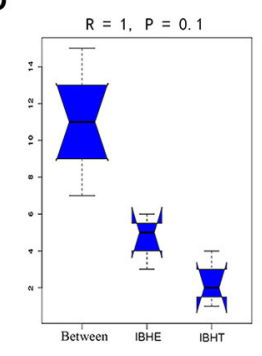

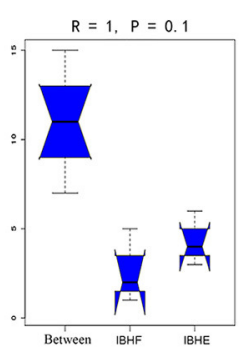

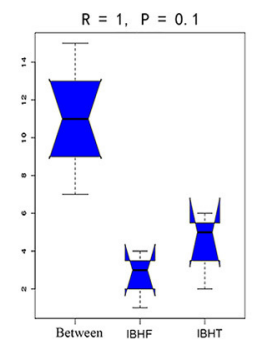

FIGURE 2 | $\beta$-Diversity analysis of samples at 8, 20, and 50 weeks. (A) Weighted uniFrac principal coordinates analysis (PCoA) of samples at 8, 20, and 50 weeks. IBHE, IBHT, and IBHF refer to "ISA Brown Hens-8 weeks," "ISA Brown Hens-20 weeks," and "ISA Brown Hens-50 weeks," respectively. Dots of different colors represent samples in different growth periods. The closer the distance between the two points, the smaller the difference of community composition. The bottom right corner of the picture (green) was IBHE, the top right corner (pink) was IBHT, and the left corner (blue) was IBHF. (B) Weighted uniFrac principal component analysis (PCA) of samples at 8, 20, and 50 weeks. In PCoA and PCA plots, abscissa and ordinate represent the first and second principal component explaining the greatest proportion of variances in the communities, respectively. (C) non-metric multidimensional scaling (NMDS) of samples at 8, 20, and 50 weeks. The stress value is used to estimate the accuracy of the model. The closer the stress value approaches zero, the better the model effect is. The model with stress value $<0.1$ can be accepted. (D) Anosim analysis of samples at 8, 20, and 50 weeks. The abscissa represents the comparison among all samples (between) and the comparison within each group, and the ordinate represents the distance between samples. The range of rank $(R)$ is -1 to $1 . R>0$ indicates that the difference between groups was greater than that within groups. $P$-value $>0.05$ indicates no statistical significance.

be clustered according to different ages, but microbial clusters became quite similar after 28 days (11).

\section{Changes of Gut Microbiota Composition in Different Growth Periods}

At the level of phyla, the predominant phyla were Firmicutes and Bacteroidetes throughout the period in this experiment, which was consistent with previous studies (6). In addition, the relative abundance of Firmicutes was higher than that of Bacteroidetes at 8 weeks in this study. It was also reported that there were around 37\% Firmicutes and 10\% Bacteroidetes in 8-week-old chickens (1). Firmicutes species are regarded as "specialists" for storage plant polysaccharides (starch and fructose) and oligosaccharides. In contrast, Bacteroidetes is a 


\section{A}

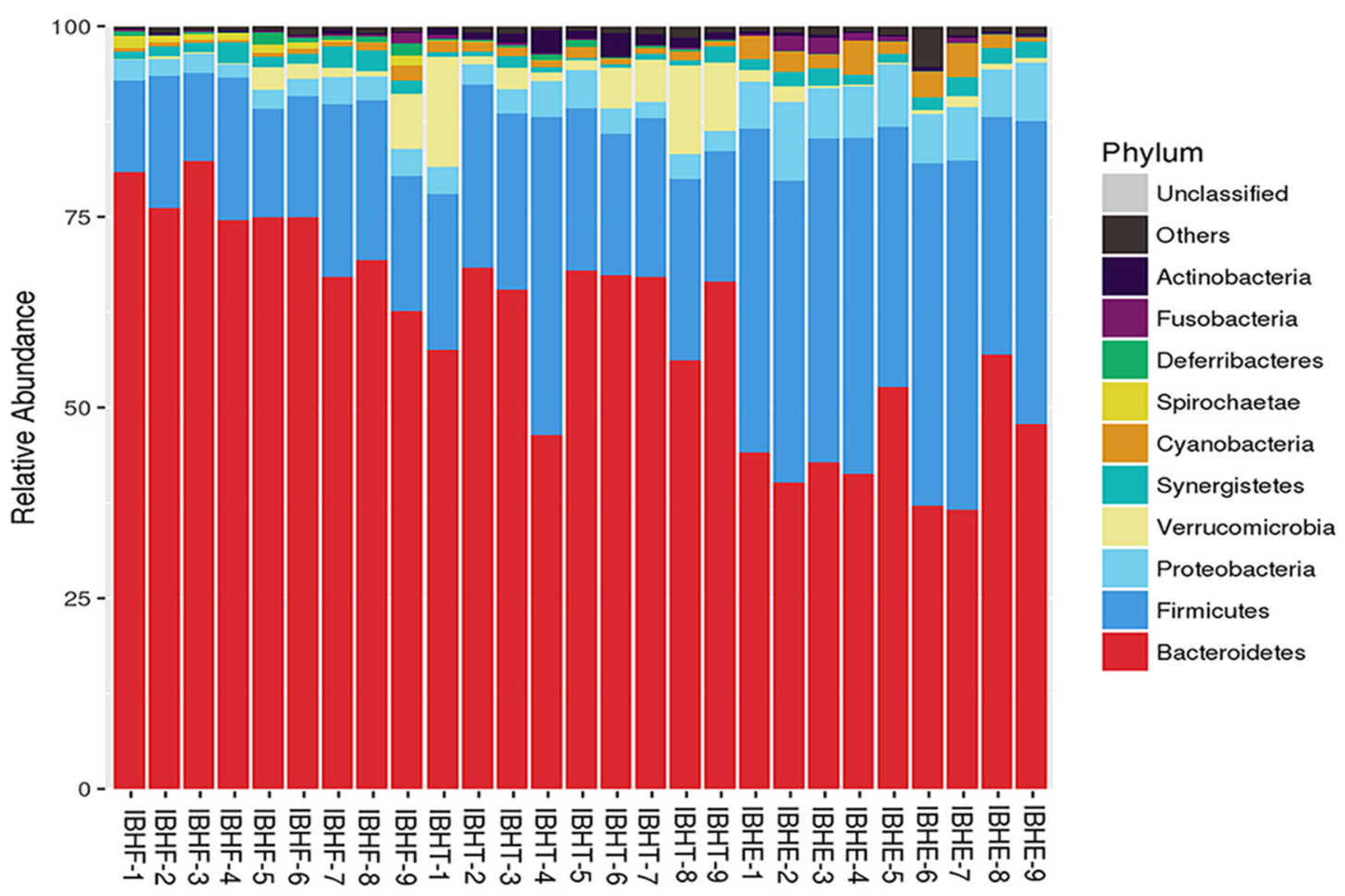

B

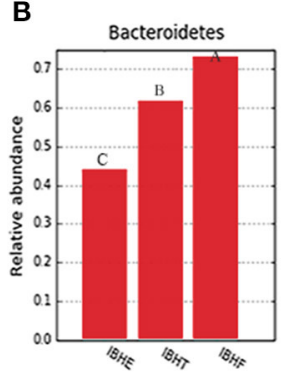

C

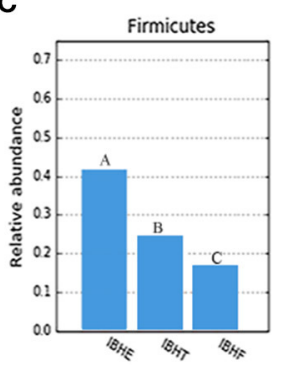

D

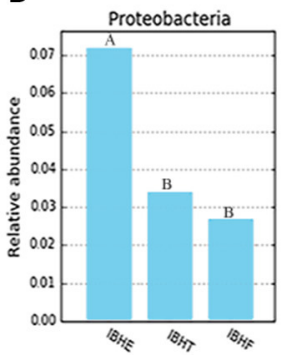

E

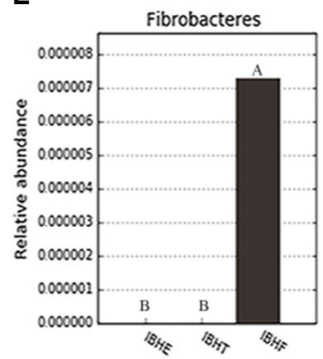

FIGURE 3 | Taxonomy stack distribution and abundance histograms of the dominant bacteria phyla of chickens in different growth periods. (A) Taxonomy stack distribution of the dominant phyla at 8, 20, and 50 weeks. IBHE, IBHT, and IBHF refer to "ISA Brown Hens-8 weeks," "ISA Brown Hens-20 weeks," and "ISA Brown Hens-50 weeks," respectively. The top 10 dominant bacteria during different periods were classified. A distinctive color histogram represents the relative distribution of these most dominant bacteria. The names of distinct levels of phyla are shown on the right of figures, and the names of samples are under the figure. (B-E) Abundance histograms of some significant dominant phyla at 8, 20, and 50 weeks. Phyla with different superscript letters mean significant differences $(P<0.05)$ between groups, and different superscript letters mean extremely significant differences $(P<0.01)$.

kind of "generalist" that degrades dietary fiber. It can utilize more a wide range of plant polysaccharides than does Firmicutes (19). In this experiment, Bacteroidetes increased from 8 to 50 weeks. This was supported by studies that reported the gradual increase of Bacteroidetes at the expense of Firmicutes during chicken rearing and egg production (18). Members of Bacteroidetes were present mainly in adult hens (44).

At the genus level, the composition of the gut microbiota in different growth periods was also different in this experiment. The dominant bacteria genus at 8 weeks did not include fiberdegradation bacteria but included the bile-tolerant bacterium
Alistipes compared with that at 20 and 50 weeks. This was maybe attributed to a high-fat and low-fiber diet (45). Alistipes specially increased in the persons who consumed animal-based diet instead of plant-based diet (46). In contrast, fiber-degradation bacteria genus Prevotellae_UCG_001 and Alloprevotella obviously increased at 20 and 50 weeks compared with 8 weeks. For 20 weeks with a high-fiber diet, this because the fiber content of it was higher than that at 8 weeks, resulting in increased fiber-degradation bacteria and Prevotellae_Ga6A1_group and Bifidobacterium. Research on human showed that the abundance of Prevotella is enriched in 

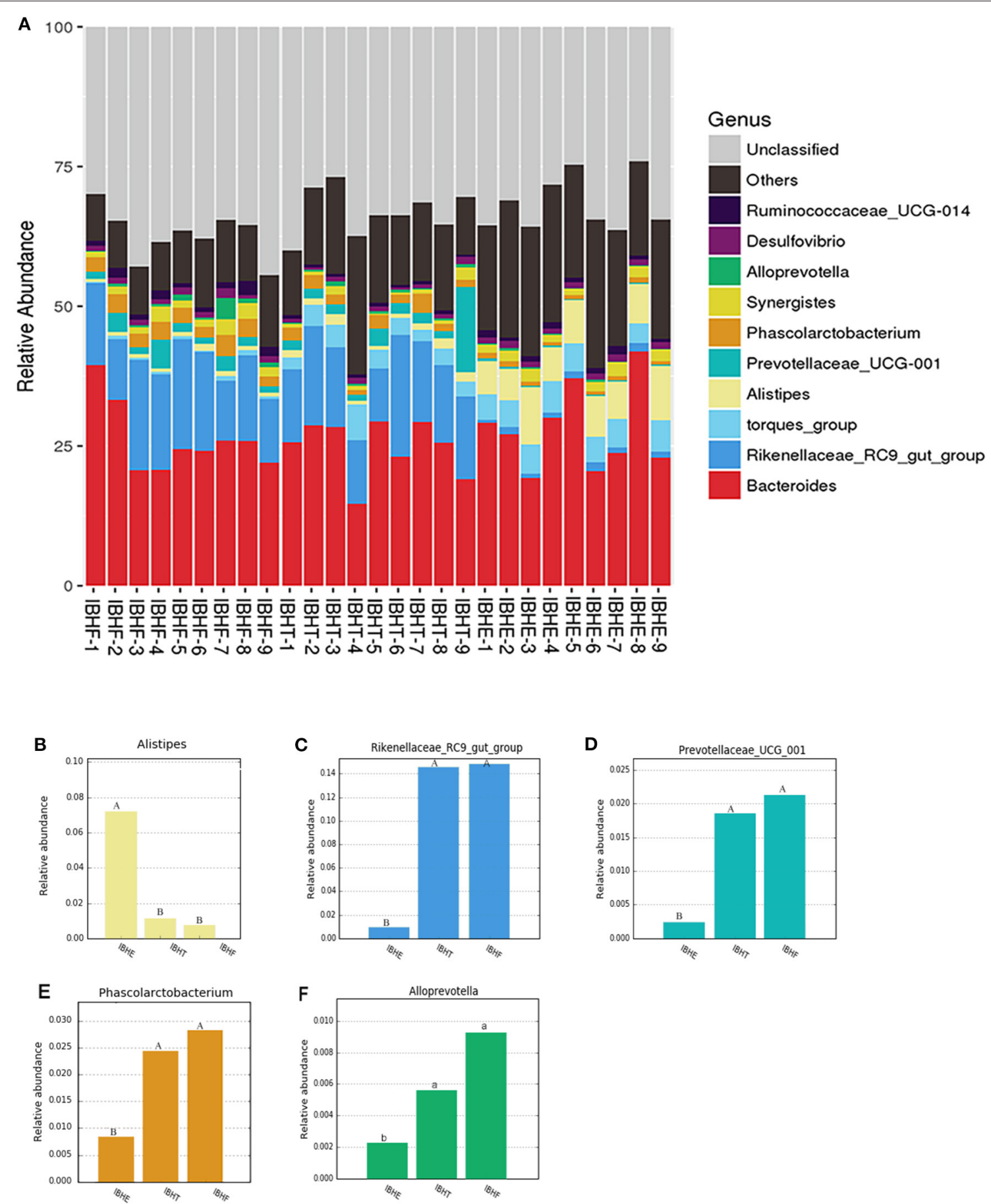

FIGURE 4 | Taxonomy stack distribution and abundance histograms of the dominant bacteria genera of chickens in different growth periods. (A) Taxonomy stack distribution of the dominant genera at 8, 20, and 50 weeks. IBHE, IBHT, and IBHF refer to "ISA Brown Hens-8 weeks," "ISA Brown Hens-20 weeks," and "ISA Brown Hens-50 weeks," respectively. The top 10 dominant bacteria genera during different periods were classified. A distinctive color histogram represents the relative distribution of these most dominant bacteria. The names of distinct levels of genera are shown on the right of the figures, and the names of the samples are under the figure. (B-F) Abundance histograms of some significant dominant genera at 8, 20, and 50 weeks. Genera with different superscript letters mean significant differences $(P<0.05)$ between groups, and different superscript letters mean extremely significant differences $(P<0.01)$.

a fiber diet (47) and has a strong ability to utilize fiber (48). Prevotella gradually became the most diverse and predominant genus with the increase of dietary fiber and age in pigs (21).
The research showed that increasing dietary fiber could increase the abundance of Bifidobacterium (49). For 50 weeks with a low-fiber diet, the feed intake was greater than that at 8 weeks, 

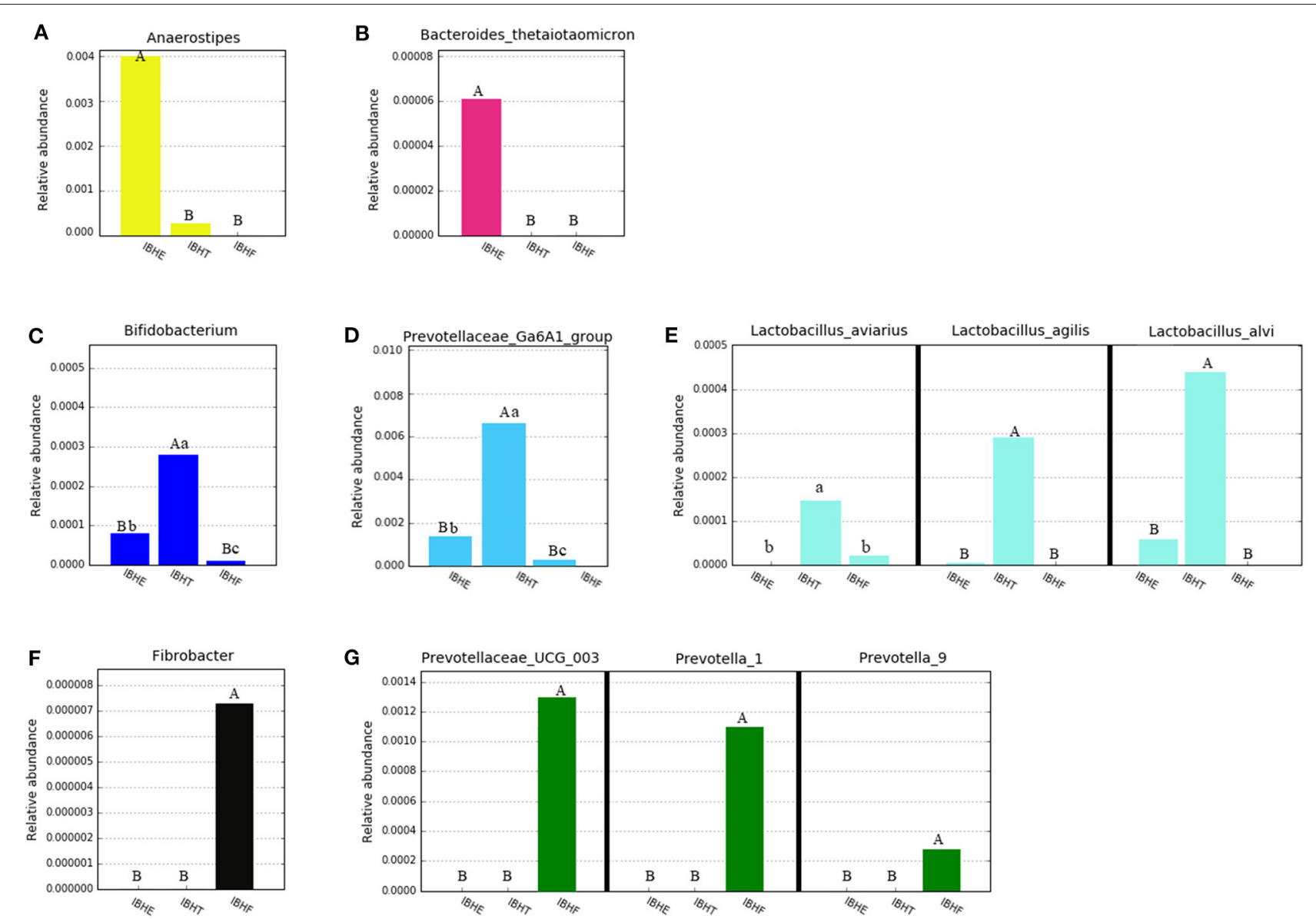

FIGURE 5 | Abundance histograms of some low-abundance dominant bacteria of chickens in different growth periods. (A,B) Abundance histograms of significant dominant short-chain fatty acid (SCFA)-producing bacteria Anaerostipes and fiber-degradation bacteria species Bacteroides thetaiotaomicron at 8 weeks. (C-E) Abundance histograms of significant dominant fiber-degradation bacteria genera Prevotellae_Ga6A1_group and Bifidobacterium and SCFA-producing species of Lactobacillus at 20 weeks. (F,G) Abundance histograms of dominant fiber-degradation bacteria genera at 50 weeks. Bacteria with different superscript letters mean significant differences $(P<0.05)$ between groups, and different superscript letters mean extremely significant differences $(P<0.01)$.

resulting in more dietary fiber being ingested. This may also help to explain why fiber-degradation bacteria Prevotella_1 and Prevotella_9 and excellent special cellulose-degradation genus Fibrobacter were only detected at 50 weeks in this experiment. Notably, as far as we know, it seems that Fibrobacter has not been reported in chickens. Fibrobacter was once believed to only exist in mammalian intestines (50), and it was first reported in the cecum of birds (ostrich) in 2010 (51).

\section{Function Annotation of Gut Microbiota in Different Growth Periods}

Software packages such as Tax4Fun can be used to predict the functional profiles of OTUs using 16S rRNA gene sequences based on the SILVA rRNA database (52). In this experiment, functional annotation of the gut microbiota showed that $\mathrm{ABC}$ transporters decreased at 8, 20, and 50 weeks. This was related to the dominant phylum, which was Firmicutes, decreasing during this period. Firmicutes has gram-positive polysaccharide utilization loci (PULs) (gpPULs), which encode
$\mathrm{ABC}$ transporters and other transporters to introduce small sugar into the periplasm for processing $(15,53)$. ABC transporter is a type of transport ATPase on the bacterial plasma membrane, and it transfers glucose to the other side of the membrane through the change of conformation. Consistently, the energy metabolism pathway and amino acid metabolism pathway were rich at 8 weeks in this study. Research also reported that the microbiota in the hindgut of 42-day-old chickens were enriched in amino acids metabolism and energy metabolism according to KEGG functional analysis (11).

In contrast, functional annotation of OTUs included a TCS; and $\beta$-glycosidase, carbohydrate metabolism pathway, and TCS pathway increased at 8,20 , and 50 weeks. This was related to the fact that the abundance of Bacteroidetes increased with age. Contrary to the gpPULs of Firmicutes, Bacteroidetes can utilize a series of plant-derived dietary polysaccharides via unique PUL $(54,55)$. PUL has been identified in all members of Bacteroidetes such as B. thetaiotaomicron and Bacteroides ovatus (19). PUL encodes a hybrid TCS, extracellular glycoside hydrolase such as 


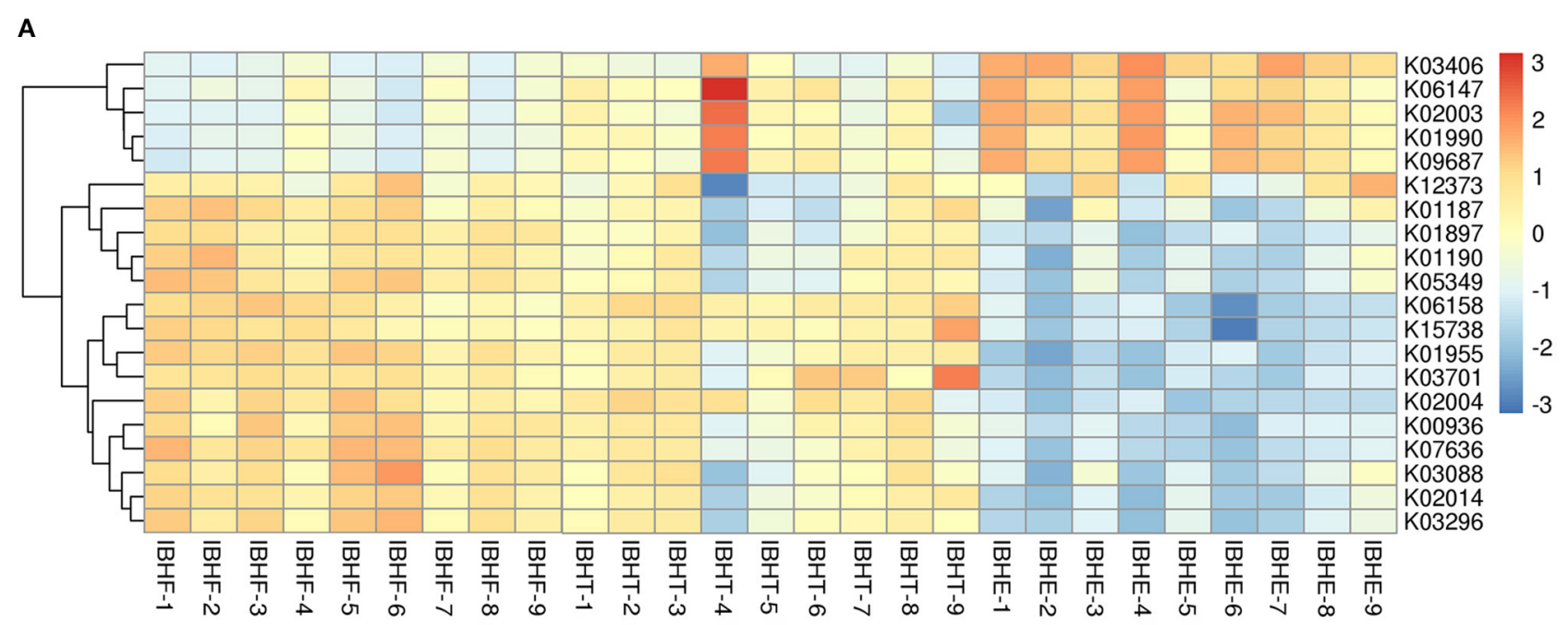

B

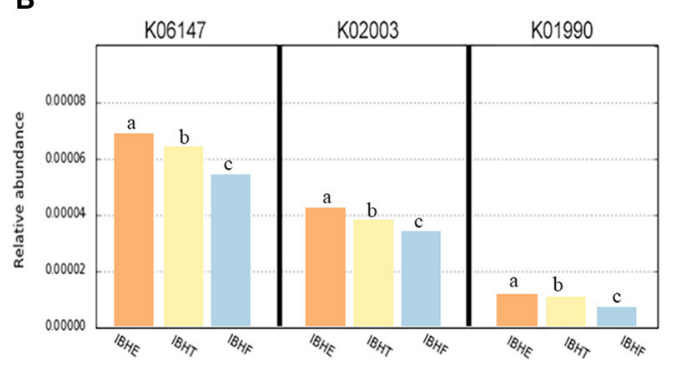

C

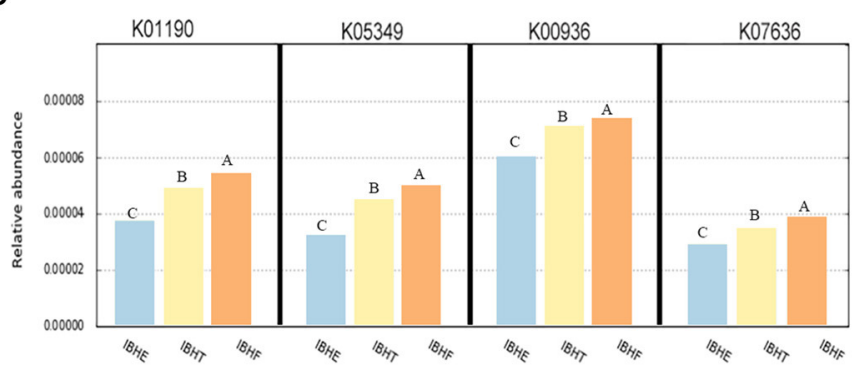

FIGURE 6 | (A) The heatmap of functional prediction of Kyoto Encyclopedia of Genes and Genomes (KEGG) Orthology (KO) of operational taxonomic units (OTUs) in different growth periods. The color gradient of heatmap was from red to yellow to blue, which indicates that the KO abundance predicted by OTU in each sample decreased gradually. The redder the color, the more bacteria performed this function. The bluer the color, the lesser bacteria performed this function. IBHE, IBHT, and IBHF refer to "ISA Brown Hens-8 weeks," "ISA Brown Hens-20 weeks," and "ISA Brown Hens-50 weeks," respectively. (B) The abundance histograms of significantly reduced KO of OTUs from 8 to 20 and 50 weeks. (C) The abundance histograms of significantly increased KO of OTUs from 8 to 20 and 50 weeks. KO with different superscript letters means significant differences $(P<0.05)$ between groups, and different superscript letters mean extremely significant differences $(P<0.01)$.

$\beta$-glycosidase (56), and other enzymes to degrade dietary fiber by cleaving glycosidic bonds (19). Consistently, carbohydrate metabolism pathway was increased with age in this experiment.

\section{The Relation Between Short-Chain Fatty Acids and Microorganisms in Different Growth Periods}

SCFAs are the major fermented metabolites of dietary fiber for SCFA-producing bacteria; primarily acetate, propionate, and butyrate account for $90-95 \%$ (57). SCFAs are very important for the growth and health of host.

In this study, the concentration of SCFAs at 20 weeks was higher than that at 8 weeks. This may be attributed to the level of fiber increase, and the numbers of some dominant fiber-degradation bacteria and SCFA-producing bacteria were more in 20 weeks. The dominant fiberdegradation bacteria at 20 weeks such as Prevotellae_UCG_001 and Alloprevotella broke down dietary fiber into more monosaccharides, and then dominant SCFA-producing bacteria Phascolarctobacterium, Bifidobacterium, and Lactobacillus fermented monosaccharides into more SCFAs than those at 8 weeks. Phascolarctobacterium can ferment monosaccharide into propionate (58), and Bifidobacterium produces acetate using "bifid-shunt" (59). Members of Lactobacillus use dietary fiber via $A B C$ transporters to produce acetate (60), which can be further fermented into butyrate by some butyrate-producing bacteria (61).

In addition, the concentration of SCFAs at 20 weeks was also greater than that at 50 weeks in this experiment. Given that the relative abundance of the dominant SCFA-producing bacteria showed no great difference between them, we speculated that it was more likely that although the microbial $\alpha$-diversity including $\mathrm{ACE}$ and Chaol at 50 weeks was higher than that at 20 weeks, most of the bacteria were not fiber-degradation bacteria or SCFA-producing bacteria; this means that more bacteria compete for limited glucose as the carbon for growth and that less glucose was fermented into SCFAs by few SCFAproducing bacteria. This may also help to explain why obese people have less microbial diversity but have more SCFAs than lean people (62). 


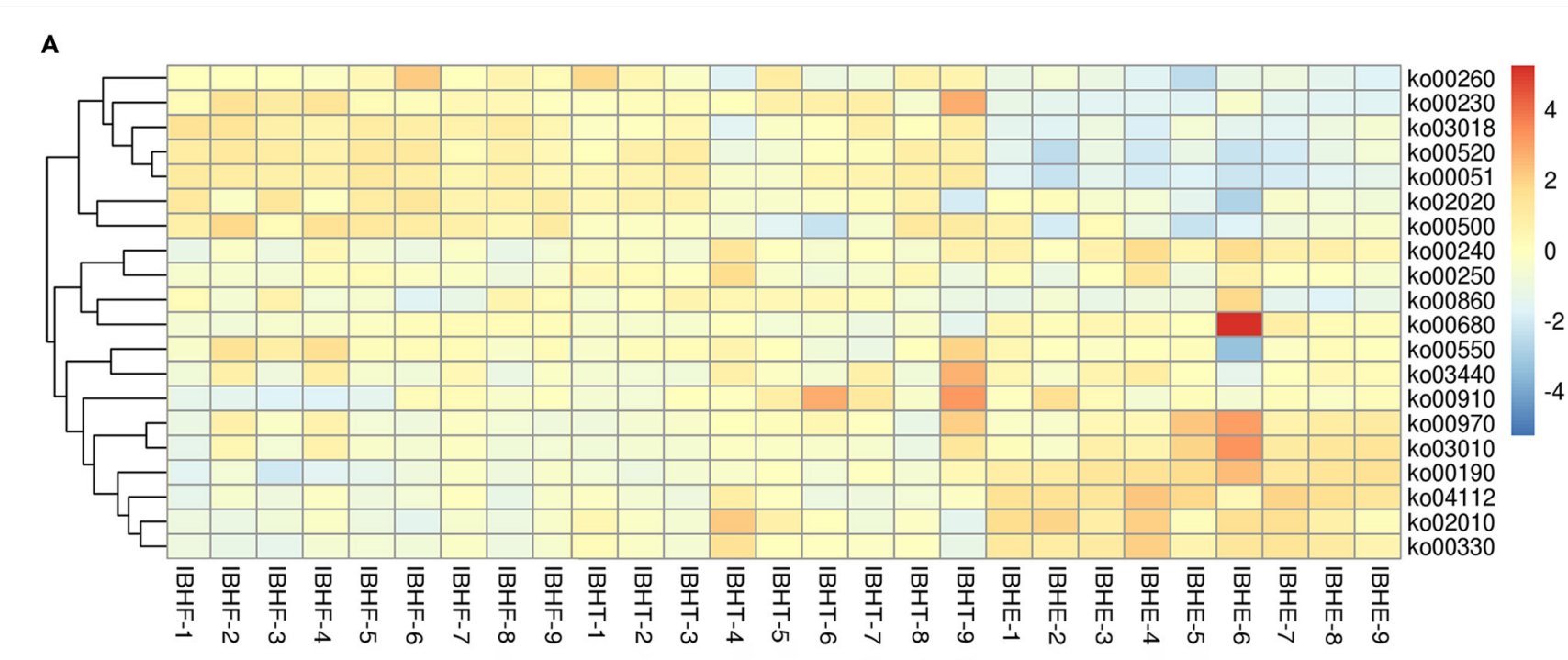

B

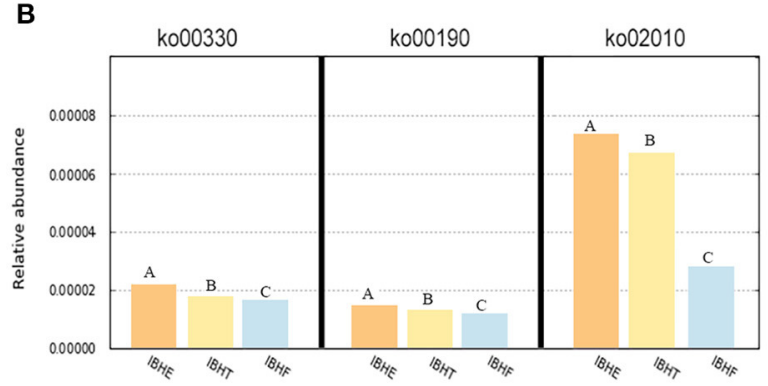

C

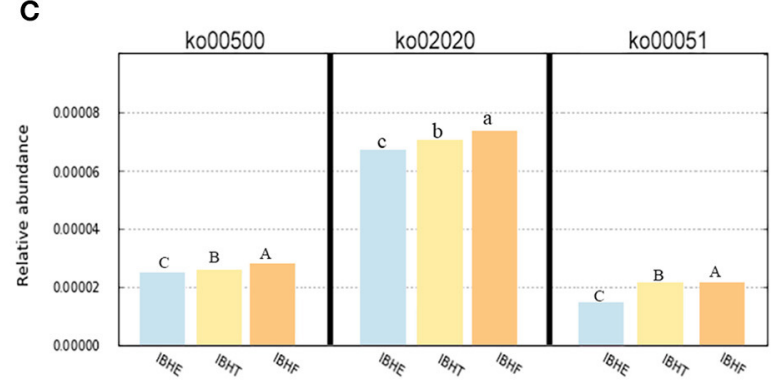

FIGURE 7 | (A) The heatmap of functional prediction of enriched metabolic pathways (ko) of OTUs in different growth periods. The color gradient of heatmap was from red to yellow to blue, which indicates that the metabolic pathway abundance predicted by OTUs in each sample decreased gradually. The redder the color, the more bacteria performed this function. The bluer the color, the lesser bacteria performed this function. IBHE, IBHT, and IBHF refer to "ISA Brown Hens-8 weeks," "ISA Brown Hens-20 weeks," and "ISA Brown Hens-50 weeks," respectively. (B) The abundance histograms of significantly increased metabolic pathways of OTUs from 8 to 20 and 50 weeks. (C) The abundance histograms of significantly reduced metabolic pathways of OTUs from 8 to 20 and 50 weeks. Metabolic pathways with different superscript letters mean significant differences $(P<0.05)$ between groups, and different superscript letters mean extremely significant differences $(P<0.01)$.

TABLE 4 | The concentration of short-chain fatty acids (SCFAs) (mmol/L) of chickens in different growth periods.

\begin{tabular}{llllll}
\hline SCFA & $\mathbf{8}$ weeks & 20 weeks & $\mathbf{5 0}$ weeks & SEM & $\boldsymbol{P}$-value \\
\hline Acetate & $2.42^{\mathrm{Bc}}$ & $6.12^{\mathrm{Aa}}$ & $3.71^{\mathrm{Bb}}$ & 0.32 & 0.006 \\
Propionate & $0.62^{\mathrm{Bb}}$ & $1.91^{\mathrm{Aa}}$ & $0.99^{\mathrm{Bb}}$ & 0.06 & 0.009 \\
Butyrate & $0.19^{\mathrm{Bb}}$ & $0.60^{\mathrm{Aa}}$ & $0.25^{\mathrm{Bb}}$ & 0.021 & 0.005
\end{tabular}

Means within a row lacking a common lowercase superscript letter mean significant differences with a $P$-value $<0.05$, and means within a row lacking a common uppercase superscript letter mean extremely significant differences with a P-value $<0.01$. Data are expressed as the means and pooled standard error of the mean (SEM).

\section{CONCLUSIONS}

The diversity, composition, and function of the gut microbiota of chickens were distinct in different growth periods. The relative abundance of the bile-acid resistant bacteria Alistipes was higher at 8 weeks compared with 20 and 50 weeks. Fiberdegradation bacteria Prevotellae_UCG_001 and Alloprevotella and SCFA-producing bacteria Phascolarctobacterium increased at 20 and 50 weeks compared with 8 weeks. In addition, $\mathrm{ABC}$ transporters decreased from 8 to 50 weeks; it might because the abundance of Firmicutes-which includes gpPULsdecreased with age. In contrast, the TCS, glucosidase, and carbohydrate metabolism pathway gradually increased from 8 to 50 weeks, because the abundance of Bacteroideteswhich includes PULs-increased with age. The concentration of SCFAs in the cecum at 20 weeks was higher than 8 and 50 weeks.

\section{DATA AVAILABILITY STATEMENT}

The datasets presented in this study can be found in online repositories. The names of the repository/repositories and accession number(s) can be found below: NCBI SRA; PRJNA701972. 


\section{ETHICS STATEMENT}

The animal study was reviewed and approved Experiment Ethics Committee (the license number: SXAU-EAW-2017-002Chi.001).

\section{AUTHOR CONTRIBUTIONS}

YY: conceptualization, resources, supervision, and funding acquisition. LH: methodology and visualization. BS and LH: formal analysis, investigation, and data curation. BS: writing-original draft preparation and review and editing.

\section{REFERENCES}

1. Zhao L, Wang G, Siegel P, He C, Wang H, Zhao W, et al. Quantitative genetic background of the host influences gut microbiomes in chickens. Sci Rep. (2013) 3:1163. doi: 10.1038/srep01163

2. van der Wielen PWJJ, Keuzenkamp DA, Lipman LJA, van Knapen F, Biesterveld S. Spatial and temporal variation of the intestinal bacterial community in commercially raised broiler chickens during growth. Microb Ecol. (2012) 44:286-93. doi: 10.1007/s00248-002-2015-y

3. Zhu X, Zhong T, Pandya Y, Joerger RD. 16S rRNA-based analysis of microbiota from the cecum of broiler chickens. Appl Environ Microbiol. (2002) 68:124-37. doi: 10.1128/AEM.68.1.124-137.2002

4. Palmer C, Bik EM, DiGiulio DB, Relman DA, Brown PO. Development of the human infant intestinal microbiota. PLoS Biol. (2007) 5:e177. doi: 10.1371/journal.pbio.0050177

5. Mackie RI, Sghir A, Gaskins HR. Developmental microbial ecology of the neonatal gastrointestinal tract. Am J Clin Nutr. (1999) 69:103545. doi: 10.1093/ajcn/69.5.1035s

6. De Maesschalck C, Eeckhaut V, Maertens L, De Lange L, Marchal L, Nezer C, et al. Gut metagenomic analysis reveals prominent roles of Lactobacillus and cecal microbiota in chicken feed efficiency. Sci Rep. (2017) 7:45308. doi: 10.1038/srep45308

7. Oakley BB, Buhr RJ, Ritz CW, Kiepper BH, Berrang ME, Seal BS, et al. Successional changes in the chicken cecal microbiome during 42 days of growth are independent of organic acid feed additives. BMC Vet Res. (2014) 10:282. doi: 10.1186/s12917-014-0282-8

8. Ballou AL, Ali RA, Mendoza MA, Ellis JC, Hassan HM, Croom WJ, et al. Development of the chick microbiome: how early exposure influences future microbial diversity. Front Vet Sci. (2016) 3:2. doi: 10.3389/fvets.2016.00002

9. Oakley BB, Kogut MH. Spatial and temporal changes in the broiler chicken cecal and fecal microbiomes and correlations of bacterial taxa with cytokine gene expression. Front Vet Sci. (2016) 3:11 doi: 10.3389/fvets.2016.00011

10. Richards P, Fothergill J, Bernardeau M, Wigley P. Development of the cecal microbiota in three broiler breeds. Front Vet Sci. (2019) 6:201. doi: 10.3389/fvets.2019.00201

11. Huang $\mathrm{P}$, Zhang Y, Xiao K, Jiang F, Wang H, Tang D, et al. The chicken gut metagenome and the modulatory effects of plant-derived benzylisoquinoline alkaloids. Microbiome. (2018) 6:211. doi: 10.1186/s40168-018-0590-5

12. Hu Y, Wang L, Shao D, Wang Q, Wu Y, Han Y, et al. Selectived and reshaped early dominant microbial community in the cecum with similar proportions and better homogenization and species diversity due to organic acids as AGP alternatives mediate their effects on broilers growth. Front Microbiol. (2020) 10:2948. doi: 10.3389/fmicb.2019. 02948

13. Han Z, Willer T, Pielsticker C, Gerzova L, Rychlik I, Rautenschlein S. Differences in host breed and diet influence colonization by and induction of local immune responses in chicken. Gut Pathog. (2016) 8:56. doi: 10.1186/s13099-0160133-1
YY and BS: project administration. All authors contributed to the article and approved the submitted version.

\section{FUNDING}

This research was funded by Construction of Key Discipline in Animal Husbandry of 1331 Engineering in Shanxi Province (J202011315), Technical System of Modern Agricultural Chicken Industry of Shanxi Province (20201102), Research and Application of Healthy Functional Animal Products Production Technology (201703D211001-05), and Key Research and Development Project of Science and Technology (Agriculture) of Jinzhong City (Y182011).

14. Kers JG, Velkers FC, Fischer EAJ, Hermes GDA, Stegeman JA, Smidt H. Host and environmental factors affecting the intestinal microbiota in chickens. Front Microbiol. (2018) 9:235. doi: 10.3389/fmicb.2018.00235

15. Polansky O, Sekelova Z, Faldynova M, Sebkova A, Sisak F, Rychlik I. Important metabolic pathways and biological processes expressed by chicken cecal microbiota. Appl Environ Microbiol. (2015) 82:156976. doi: 10.1128/AEM.03473-15

16. Shabbir MZ, Malys T, Ivanov YV, Park J, Shabbir MA, Rabbani M, et al. Microbial communities present in the lower respiratory tract of clinically healthy birds in Pakistan. Poult Sci. (2015) 94:612-20. doi: 10.3382/ps/pev010

17. Ngunjiri JM, Taylor KJM, Abundo MC, Jang H, Elaish M, Mahesh KC, et al. Farm stage, bird age, and body site dominantly affect the quantity, taxonomic composition, and dynamics of respiratory and gut microbiota of commercial layer chickens. Appl Environ Microbiol. (2019) 85:e0313718. doi: 10.1128/AEM.03137-18

18. Videnska P, Rahman MM, Faldynova M, Babak V, Matulova ME, et al. Characterization of egg laying hen and broiler fecal microbiota in poultry farms in croatia, czech republic, hungary and slovenia. PLOS ONE. (2014) 9:e110076. doi: 10.1371/journal.pone.0110076

19. Martens EC, Lowe EC, Chiang H, Pudlo NA, Gordon JI, et al. Recognition and degradation of plant cell wall polysaccharides by two human gut symbionts. PLoS Biol. (2011) 9:e1001221. doi: 10.1371/journal.pbio.1001221

20. Luis AS, Briggs J, Zhang X, Farnell B, Ndeh D, Labourel A, et al. Dietary pectic glycans are degraded by coordinated enzyme pathways in human colonic Bacteroides. Nat Microbiol. (2017) 3:210-9. doi: 10.1038/s41564-017-0079-1

21. Wang X, Tsai T, Deng F, Wei X, Chai J, Knapp J, et al. Longitudinal investigation of the swine gut microbiome from birth to market reveals stage and growth performance associated bacteria. Microbiome. (2019) 7:1. doi: 10.1186/s40168-019-0721-7

22. Rakoff-Nahoum S, Kevin RF, Laurie EC. The evolution of cooperation within the gut microbiota. Nature. (2016) 533:255-9. doi: 10.1038/nature17626

23. Leth ML, Ejby M, Workman C, Ewald DA, Pedersen SS, Sternberg C, et al. Differential bacterial capture and transport preferences facilitate cogrowth on dietary xylan in the human gut. Nat Microbiol. (2018) 3:57080. doi: 10.1038/s41564-018-0132-8

24. Dunkley KD, Dunkley CS, Njongmeta NL, Callaway TR, Hume ME, Kubena LF, et al. Comparison of in vitro fermentation and molecular microbial profiles of high-fiber feed substrates incubated with chicken cecal inocula. Poult Sci. (2007) 86:801-10. doi: 10.1093/ps/86.5.801

25. van Der Wielen PW, Biesterveld S, Notermans S, Hofstra H, Urlings BA, van Knapen. F. Role of volatile fatty acids in development of the cecal microflora in broiler chickens during growth. Appl Environ Microbiol. (2000) 66:2536-40. doi: 10.1128/AEM.66.6.2536-2540.2000

26. Valentina T, Fredrik B. Functional interactions between the gut microbiota and host metabolism. Nature. (2012) 489:242-9. doi: 10.1038/nature11552

27. Macfarlane S, Macfarlane GT. Regulation of short-chain fatty acid production. Proc Nutr Soc. (2003) 62:67-72. doi: 10.1079/PNS2002207

28. Brown AJ, Goldsworthy SM, Barnes AA, Eilert MM, Tcheang L, Daniels D, et al. The orphan G protein-coupled receptors GPR41 and GPR43 are activated 
by propionate and other short chain carboxylic acid. J Biol Chem. (2003) 27:11312-9. doi: 10.1074/jbc.M211609200

29. Callaway TR, Dowd SE, Wolcott RD, Sun Y, McReynolds JL, et al. Evaluation of the bacterial diversity in cecal contents of laying hens fed various molting diets by using bacterial tag-encoded FLX amplicon pyrosequencing. Poult Sci. (2009) 88:298-302. doi: 10.3382/ps.2008-00222

30. Magoč T, Salzberg SL. FLASH: fast length adjustment of short reads to improve genome assemblies. Bioinformatics. (2011) 27:2957-63. doi: 10.1093/bioinformatics/btr507

31. Caporaso JG, Kuczynski J, Stombaugh J, Bittinger K, Bushman FD, Costello EK, et al. QIIME allows analysis of high-throughput community sequencing data. Nat Methods. (2010) 7:335-6. doi: 10.1038/nmeth.f.303

32. Bokulich NA, Subramanian S, Faith JJ, Gevers D, Gordon JI, Knight R, et al. Quality-filtering vastly improves diversity estimates from Illumina amplicon sequencing. Nat Methods. (2013) 10:57-9. doi: 10.1038/nmeth.2276

33. Edgar RC. UPARSE, highly accurate OTU sequences from microbial amplicon reads. Nat Methods. (2013) 10:996-8. doi: 10.1038/nmeth.2604

34. Wang Q, Garrity GM, Tiedje JM, Cole JR. Naive Bayesian classifier for rapid assignment of rRNA sequences into the new bacterial taxonomy. Appl Environ Microbiol. (2007) 73:5261-7. doi: 10.1128/AEM.00062-07

35. Prüsse E, Quast C, Yilmaz P, Ludwig W, Peplies J, Glöckner FO. SILVA, a comprehensive online resource for quality checked and aligned ribosomal RNA sequence data compatible with ARB. Nucleic Acids Res. (2007) 35:718896. doi: 10.1093/nar/gkm864

36. Ondov $\mathrm{BD}$, Bergman $\mathrm{NH}$, Phillippy AM. Interactive metagenomic visualization in a Web browser. BMC Bioinform. (2011) 12:385. doi: 10.1186/1471-2105-12-385

37. Oksanen J, Blanchet FG, Kindt R, Legendre P, O’Hara RB, Simpson GL, et al. Vegan, community ecology package. $\mathrm{R}$ package version 1.17-4. Acesso 23 (2010).

38. Edgar RC. MUSCLE, multiple sequence alignment with high accuracy and high throughput. Nucleic Acids Res. (2004) 32:1792-7. doi: 10.1093/nar/gkh340

39. Aßhauer KP, Wemheuer B, Daniel R, Meinicke P. Tax4Fun, predicting functional profiles from metagenomic 16S rRNA data. Bioinformatics. (2015) 31:2882-4. doi: 10.1093/bioinformatics/btv287

40. Hou L, Sun B, Yang Y. Effects of added dietary fiber and rearing system on the gut microbial diversity and gut health of chicken. Animals. (2020) 10:1-22. doi: 10.3390/ani10010107

41. White JR, Nagarajan N, Pop M. Statistical methods for detecting differentially abundant features in clinical metagenomic samples. PLoS Comput Biol. (2009) 5:e1000352. doi: 10.1371/journal.pcbi.1000352

42. Brisbin JT, Gong J, Sharif S. Interactions between commensal bacteria and the gut-associated immune system of the chicken. Anim Health Res Rev. (2008) 9:101-10. doi: 10.1017/S146625230800145X

43. Lu J, Idris U, Harmon B, Hofacre C, Maurer JJ, Lee MD. Diversity and succession of the intestinal bacterial community of the maturing broiler chicken. Appl Environ Microbiol. (2003) 69:6816-24. doi: 10.1128/AEM.69.11.6816-6824.2003

44. Videnska P, Sedlar K, Lukac M, Faldynova M, Gerzova L, Cejkova $D$, et al. Succession and replacement of bacterial populations in the caecum of egg laying hens over their whole life. PLoS ONE. (2014) 9:e0115142. doi: 10.1371/journal.pone.0115142

45. Zhang C, Zhang M, Pang X, Zhao Y, Wang L, Zhao L. Structural resilience of the gut microbiota in adult mice under high-fat dietary perturbations. ISME J. (2012) 6:1848-57. doi: 10.1038/ismej.2012.27

46. David LA, Maurice CF, Carmody RN, Gootenberg DB, Button JE, Wolfe BE, et al. Diet rapidly and reproducibly alters the human gut microbiome. Nature. (2014) 505:559-77. doi: 10.1038/nature12820

47. Paola MD, Filippo CD, Cavalieri D, Ramazzotti M, Poullet JB, Massart S, et al. Impact of diet in shaping gut microbiota revealed by a comparative study in children from Europe and rural Africa. PNAS. (2010) 107:146916. doi: $10.1073 /$ pnas. 1005963107
48. Gorvitovskaia A, Holmes SP, Huse SM. Interpreting Prevotella and Bacteroides as biomarkers of diet and lifestyle. Microbiome. (2016) 4:15. doi: 10.1186/s40168-0160160-7

49. Zhao L, Zhang F, Ding X, Wu G, Lam YY, Wang X, et al. Gut bacteria selectively promoted by dietary fibers alleviate type 2 diabetes. Science. (2018) 359:1151-6. doi: 10.1126/science.aao5774

50. Ransom-Jones E, Jones DL, McCarthy AJ, McDonald JE. The Fibrobacteres: an important phylum of cellulose-degrading bacteria. Microb Ecol. (2012) 63:267-81. doi: 10.1007/s00248-011-9998-1

51. Matsui H, Kato Y, Chikaraishi T, Moritani M, Ban-Tokuda T, Wakita M. Microbial diversity in ostrich ceca as revealed by $16 \mathrm{~S}$ ribosomal RNA gene clone library and detection of novel Fibrobacter species. Anaerobe. (2010) 16:83-93. doi: 10.1016/j.anaerobe.2009.07.005

52. Langille MGI, Zaneveld J, Caporaso JG, McDonald D, Knights D, Reyes JA, et al. Predictive functional profiling of microbial communities using 16S rRNA marker gene sequences. Nat Biotechnol. (2013) 31:81421. doi: $10.1038 /$ nbt.2676

53. Sheridan PO, Martin JC, Lawley TD, Browne HP, Harris HMB, BernalierDonadille A, et al. Polysaccharide utilization loci and nutritional specialization in a dominant group of butyrate-producing human colonic Firmicutes. Microb Genom. (2016) 2:e000043. doi: 10.1099/mgen.0.000043

54. Larsbrink J, Rogers TE, Hemsworth GR, McKee LS, Tauzin AS, Spadiut $\mathrm{O}$, et al. A discrete genetic locus confers xyloglucan metabolism in select human gut Bacteroidetes. Nature. (2014) 506:498-502. doi: 10.1038/nature 12907

55. Accetto T, Avguštin G. Polysaccharide utilization locus and CAZYme genome repertoires reveal diverse ecological adaptation of Prevotella species. Syst Appl Microbiol. (2015) 38:453-61. doi: 10.1016/j.syapm.2015.07.007

56. D'Elia JN, Salyers AA. Effect of regulatory protein levels on utilization of starch by Bacteroides thetaiotaomicron. J Bacteriol. (1996) 178:71806. doi: $10.1128 /$ jb.178.24.7180-7186.1996

57. Jozefiak D, Rutkowski A, Martin S. Carbohydrate fermentation in the avian ceca: a review. Anim Feed Sci Technol. (2004) 113:1-15. doi: 10.1016/j.anifeedsci.2003.09.007

58. Hilpert W, Dimroth P. Conversion of the chemical energy of methylmalonylCoA decarboxylation into a $\mathrm{Na}+$ gradient. Nature. (1982) 296:5845. doi: $10.1038 / 296584 \mathrm{a} 0$

59. Derrien M, van Hylckama Vlieg JET. Fate, activity, and impact of ingested bacteria within the human gut microbiota. Trends Microbiol. (2015) 23:35466. doi: $10.1016 /$ j.tim.2015.03.002

60. Barrangou R, Altermann E, Hutkins $R$ Cano, Klaenhammer $T R$. Functional and comparative genomic analyses of an operon involved in Fructooligosaccharide utilization by Lactobacillus acidophilus. Proc Natl Acad Sci USA. (2003) 100:8957-62. doi: 10.1073/pnas. 1332765100

61. Louis P, Young P, Holtrop G, Flint HJ. Diversity of human colonic butyrate-producing bacteria revealed by analysis of the butyrylCoA:acetate CoA-transferase gene. Environ Microbiol. (2010) 12:304-14. doi: 10.1111/j.1462-2920.2009.02066.x

62. Cho I, Yamanishi S, Cox L, Methé BA, Zavadil J, Li K, et al. Antibiotics in early life alter the murine colonic microbiome and adiposity. Nature. (2012) 488:621-6. doi: 10.1038/nature11400

Conflict of Interest: The authors declare that the research was conducted in the absence of any commercial or financial relationships that could be construed as a potential conflict of interest.

Copyright (๑) 2021 Sun, Hou and Yang. This is an open-access article distributed under the terms of the Creative Commons Attribution License (CC BY). The use, distribution or reproduction in other forums is permitted, provided the original author(s) and the copyright owner(s) are credited and that the original publication in this journal is cited, in accordance with accepted academic practice. No use, distribution or reproduction is permitted which does not comply with these terms. 